\title{
ANALYSIS OF VEHICLE BOGIE EFFECTS ON TRACK STRUCTURE - NON-STATIONARY ANALYSIS OF DYNAMIC RESPONSE
}

The present paper is concentrated on the analysis of in situ vibration measurements during operating conditions in the track structure for loading by current passenger trains at the new built up railway corridor in the section Bratislava - Trnava. Tests were carried out to determine the dynamic track behaviour and relative dynamic effects between the track components - the rails, the sleepers, and the ballast bed during the passage of characteristic passenger trains moving at the operating speeds $70 \div 130 \mathrm{~km} / \mathrm{h}$. Experimentally obtained signals of the dynamic response of track components - measured as vertical accelerations and displacements of the rails, sleepers, and ballast bed are analysed in detail as the nonstationary random signals corresponding passages of characteristic vehicle bogies (locomotives, coaches). Vertical dynamic response of these components were measured at two different locations of this section and the vertical dynamic behaviour of track components were investigated in the time domain and analysed in the frequency domain up to $500 \mathrm{~Hz}$.

\section{Introduction}

Dynamic phenomena in the track structure are associated with the operating conditions and are in the direct relation with the vehicle-track dynamic interaction. Field tests were carried out to determine the dynamic behaviour and relative vertical dynamic effects between the track components - the rails, the sleepers, and the ballast bed during the passage of characteristic passenger and freight trains moving at the operating speeds. In concentrating on the track structure the dynamic effects may be divided into two groups:

1. The vibration of the track structure - the vibration of rails, sleepers, and the ballast layer and the wave propagation in the track structure.

2. The wave propagation in the surrounding of the track which can produce vibrations to adjacent structures and radiated noise.

This paper is focused on the first area of the dynamic response. The dynamic phenomena in the track structure are caused by several mechanisms which are well known:

- the quasi-static excitation due to the moving axle load of trains,

- the wheel and the rail roughness and their defects,

- the type of trains loading the track and their composition and speed,

- the excitation due to discrete supports of the rail by sleepers, etc.

When the frequencies of interest are low, we speak about "structural vibration" and for higher frequency components about "structural-borne sounds" and "air-borne sounds". Their origin of sound emission, however, is always in structural vibration of track components. One effect of the moving load is associated with the wave propagation in the rails and in the subsoil which is presented at the higher speed train. Another phenomenon is linked to the Doppler effect which forms two peaks in the frequency spectrum for any frequency component.

All these dynamic phenomena are analyzed in particular through the theoretical - simulation approaches, such as in Refs. $[1,2,5,6]$. One simulation model developed at the Department of Structural Mechanics [5], was addressed to the study of dynamic behaviour of the track structure, especially the evaluation of the dynamic behaviour and the response of track components (rails, sleepers, the ballast bed) due to variability in the vertical stiffness of rail supports [5, 6]. In fewer cases the experimental approach is applied, especially focussed on the free field vibration measurements during the passage of train $[3,4,7]$. In addition to analytical models for the dynamic train-track interaction the experimental analyses are needed for the validation and appreciation of these vibrations. Experimental results have also important place in dynamic tuning of the moving vehicle - track. The three most common approaches to detect dynamic response of track components are based on the use of either:

(a) Deflections measurement $w_{x}(t)$ using the displacement transducers DR, DS imbedded in a fixed reference datum at a position $\mathrm{x}$ to measure the response, see Figs. 3, 4.

(b) Acceleration measurements $w_{x}^{\prime \prime}(t)$ using the accelerometers $x=A_{R}, A_{S}, A_{B}$ that are glued on the rail web or on the sleeper and which require no fixed datum to measure the response at a position $x$, see Figs. 3, 4 .

(c) Strain measurements $\varepsilon_{x}(t)$ using the strain gauges $T_{R}$ that are glued on the rail web (R) or on the sleeper (S) at a position $X$ to measure the response.

\footnotetext{
* Milan Moravcik

Faculty of Civil Engineering, University of Zilina, Slovakia, E-mail: milan.moravcik@fstav.uniza.sk
} 
This contribution is devoted right to experimental measurements and the detailed analyses of in situ measured signals in the frequency domain up to $500 \mathrm{~Hz}$. The paper is focused on the analysis of the track response of passenger trains only. The frequency composition of the vibration is measured as vertical accelerations $w^{\prime \prime}{ }_{x}(t)$ for track components $X$ at the position $\mathrm{x}$ on the track: $x \rightarrow$ the rail / accelerometer $A_{R}$, sleepers / the accelerometer $A_{S}$, and the ballast bed / the accelerometer $A_{B}$. Displacement amplitudes can be calculated from measured accelerations. Displacement amplitudes $w_{x}(t)$ were measured also direct by displacement transducers $D_{R}, D_{S}$.

\section{Measured track structure}

The in situ measurements were made on the newly built up railway corridor in the line Bratislava - Żilina at the two locations at the straight section track Cífer - Trnava, Fig. 1. Measurements were executed in the summer 2008 and 2009. Measured track is the convectional ballasted track with UIC 60 rails supported every $0.60 \mathrm{~m}$ by rubber pads on the monoblock concrete sleepers of the type PB-3/Cana. The rails are continuously welded and are fixed with the Vossloh fastening system Skl 14.

The track is supported by the substructure consisting of ballast layer (gravel aggregates of the calibre $32 / 53 \mathrm{~mm}$ ) of the thickness $h_{1}=0.35 \div 0.55 \mathrm{~m}$, the sub-ballast layer of the thickness $h_{2}$ - the gravel support layer $h_{2}=0.4 \mathrm{~m}$ laying on the geotextile of the type Tensar SS 30 and the filtering geotextile Tatratex T200, put on the subgrade, see Fig. 2.

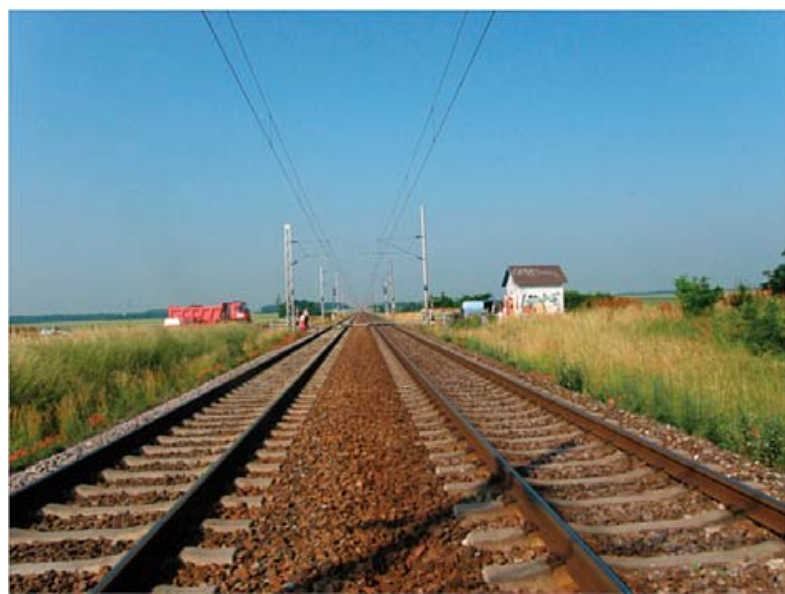

Fig. 1 The measured track section in the location Cifer - Trnava

\section{Experimental setup}

The vertical vibrations of rails (R), sleepers (S) and ballast layer (B) between the sleepers were measured as the vertical acceleration $w^{\prime \prime}{ }_{x}(t), x=(R, S, B)$, the vertical relative displacement $w_{x}(t), x=(R, S)$, and the strain measurement $\varepsilon_{x}(t)$ at a position $x$

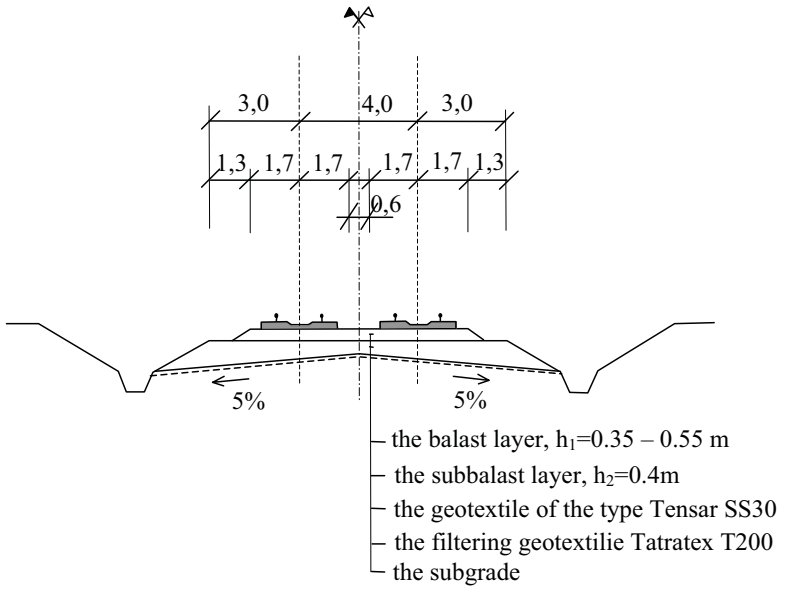

Fig. 2 The cross section of the ballasted track at the measured track section.

$=(R, S)$ on the rail or on the sleepers. The $\mathrm{B} \& \mathrm{~K}$ piezoelectric accelerometers $A_{R}, A_{S}$, of the type BK 4500 were glued to the rail and the sleeper. The BK 8306 seismic accelerometer $A_{B}$ was embedded into the plaster bed in ballast layer between the sleepers at the depth of placing sleepers, see Figs. 3 and 4 . The vertical displacements $w_{R}(t)$ of the rail and the sleeper $w_{S}(t)$ were measured by the relative displacement transducers of the type Bosh mounted on the fixed reference datum (displacement transducers $D_{R}, D_{S}$ ). The direct dynamic strain $\varepsilon(t)$ of the rail was measured by the Kistler piezoelectric tensiometer $T_{R}$ mounted on the rail flange.

Direct dynamic deflection measurements using the displacement transducers $D_{R}, D_{S}$ provide a frequency response limited. Using accelerometers $A_{R}, A_{S}, A_{B}$ gives the response in the broad frequency range and is attractive since no fix datum is required.

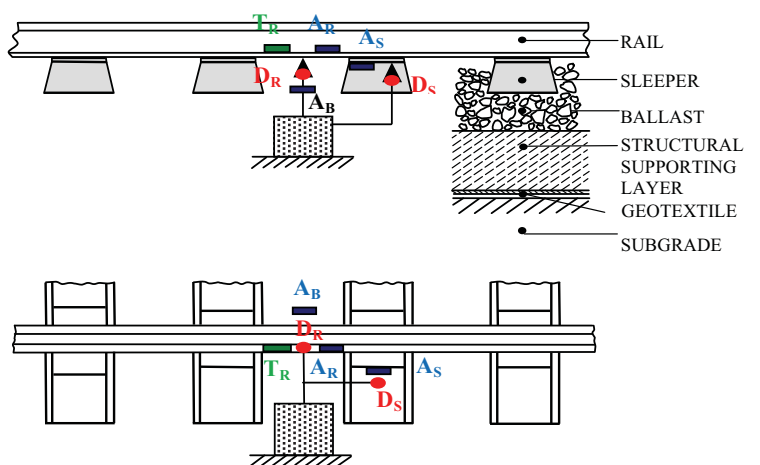

- $\mathbf{D}_{\mathrm{R}}, \mathbf{D}_{\mathrm{S}}$ : VERTICAL DISPLACEMENT TRANSDUCERS ( BOSH)

- $\mathbf{A}_{\mathrm{R}}, \mathbf{A}_{\mathrm{S}}, \mathbf{A}_{\mathrm{B}}$ : ACCELEROMETERS ( BK 4500; BK 3806)

च $\mathrm{T}_{\mathrm{R}}$ : TENSIOMETERS ( KISTLER)

Fig. 3 Scheme of the measurement set-up and positioning transducers in the track.

Measured quantities - deflections $w_{x}(t)$, vertical accelerations $w^{\prime \prime}{ }_{x}(t)$, and strains $\varepsilon_{x}(t)$ were recorded as electrical signals and 
transformed by means of the analogy-digital convector of the type 32-channel NI CompactDAQ data acquisition. The A/D device records the signals with the chosen sampling frequency $f_{s}=1000$ $\mathrm{Hz}$ directly into the computer memory. The NI DIAdem interactive software for managing and analysing the data was applied.

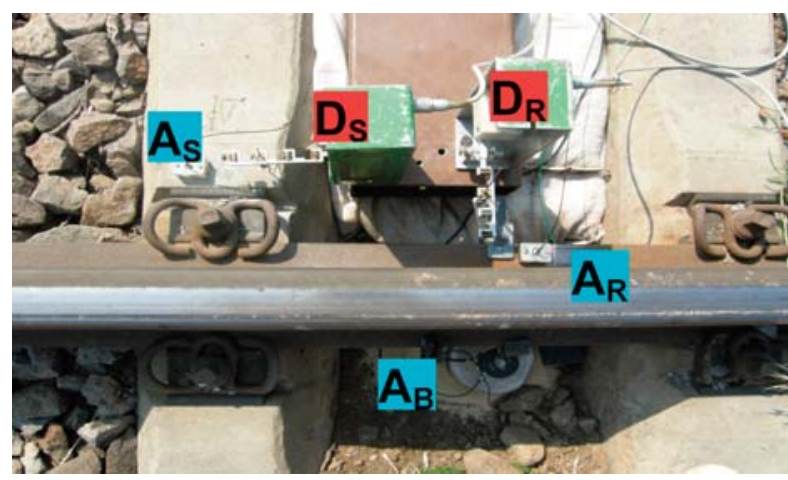

Fig. 4 Position of transducers in the track: Transducers $D_{R}, A_{R}$ on the rail, accelerometers $A_{S}, A_{S}$ on the sleeper, and $A_{B}$ in the ballast.

\section{The characteristics of the measured passenger trains}

The measured track section was loaded by the passenger and freight trains running in operational conditions. As the paper is devoted to the analysis of passenger trains only, the typical configuration of a passenger train is shown in Fig. 5. They consist of a locomotive and $5 \div 10$ carriages of a similar composition. The speed of passenger trains in the measured section varies between $90 \mathrm{~km} / \mathrm{h}$ $\div 130 \mathrm{~km} / \mathrm{h}$. The locomotives of passenger trains (types EL350, EL150, EL263) are supported by 2 bogies, equally as the coaches, see Fig. 5 and Tab. 1.

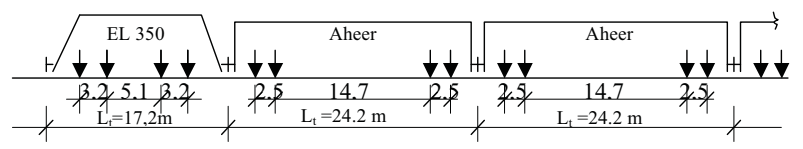

Fig. 5 A typical configuration of the measured passenger train.
The basic spatial and mass characteristics of locomotives and coaches for the passenger transport are the coach total length $L_{t}$, the distance between bogies $L_{b}$, the wheelset distance in a bogie $L_{a}$, the total vehicle mass $M_{t}$, the bogie mass $m_{\text {bog }}$, and the one axle mass (the unsprung mass of the one wheelset) $m_{1, \text { uns }}$. and they are summarised in Table 1. While the quasi-static contribution in the track response depends on the total mass of vehicles $M_{t}$, the dynamic load contributions depend dominantly on the unsprung mass of wheelsets $\mathrm{m} 1$,uns.

\section{Measured time history during the passage of trains}

In Fig. 6 are presented characteristic time histories of the rail deflection $w_{R}(t)$, the rail acceleration $w^{\prime \prime}{ }_{R}(t)$, and the sleeper acceleration $w^{\prime \prime}{ }_{S}(t)$ corresponding to the passage of the passenger train EL $350+8$ carriages, speed $c=32.9 \mathrm{~m} / \mathrm{s}=119 \mathrm{~km} / \mathrm{h}$.

\subsection{Time histories of the track response and their analysis - Nonstationary signals}

File TN II/No.2: The passage of the passenger train EL $350+8$ coaches, EL $350+8$ coaches, LABWIEV, $f_{s}=1000 \mathrm{~Hz}$, the nonfiltered time records, see Fig. 6a, b, c, d:

a/ Time history of the vertical rail displacement $w_{R}(t) /$ the transducer $D_{R}$,

b/ Time history of the vertical rail acceleration $w^{\prime \prime}{ }_{R}(t) /$ the accelerometer $A_{R}$,

c/ Time history of the vertical sleeper acceleration $w_{S}^{\prime \prime}(t)$ the accelerometer $A_{S}$,

d/ Time history of the vertical ballast bed acceleration /the accelerometer $A_{B}$.

The bogie passages of a locomotive and coaches next the locomotive form a characteristic load of the track - impulse load activated by vehicle bogies.

The obtained time histories of the vertical acceleration (measured signals) $w^{\prime \prime}{ }_{R}(t), w^{\prime \prime}{ }_{S}(t)$ and $w_{B}{ }_{B}(t)$ represent generally random functions of time $t$ describing the track response. In the full sense of the word they represent the generally nonstationary random

The geometrical and mass characteristics of vehicles for the coaching traffic in ŽSR

Table 1

\begin{tabular}{|l|c|c|c|c|c|c|c|}
\hline \multirow{2}{*}{ Locomotives } & \multirow{2}{*}{ Axles } & \multicolumn{3}{|c|}{ The length [m] } & \multicolumn{3}{c|}{ The mass [t] } \\
\cline { 3 - 8 } & & $L_{t}$ & $L_{b}$ & $L_{a}$ & $\begin{array}{c}\text { The total vehicle } \\
\text { mass } M_{t}\end{array}$ & $\begin{array}{c}\text { The bogie mass } \\
m_{\text {bog. }}\end{array}$ & $\begin{array}{c}\text { The axle mass } \\
m_{1, \text { uns. }}\end{array}$ \\
\hline EL 162, 163 & 4 & 16.80 & 5.10 & 3.20 & 85.0 & 20.520 & 2.466 \\
EL350 & 4 & 17.24 & 5.10 & 3.20 & 87.6 & 20.520 & 2.466 \\
EL 262, 363 & 4 & 16.80 & 5.10 & 3.20 & 87.0 & 20.520 & 2.466 \\
EL 150 & 4 & 16.74 & 5.10 & 3.20 & 87.0 & 20.520 & 2.466 \\
\hline Carriages & Axles & Lt & Lb & La & Mt & mbog. & m1,uns. \\
\hline Aheer, Bheer & 4 & 24.5 & 14.7 & 2.5 & $43.0 \div 48.0$ & 6.400 & 1.34 \\
Bte & 4 & 24.5 & 14.6 & 2.6 & $34.0 \div 42.0$ & 6.800 & 1.45 \\
Bai & 4 & 24.5 & 14.7 & 2.6 & $38.0 \div 44.0$ & 6.800 & 1.45 \\
\hline
\end{tabular}




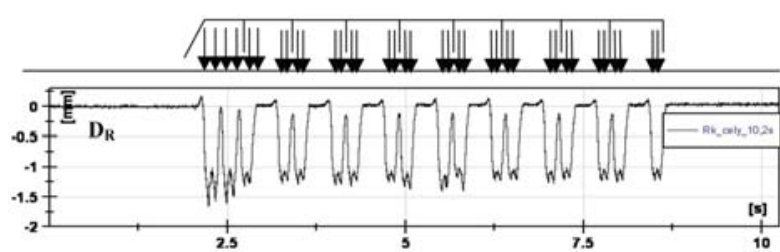

a/ Time history of the vertical rail displacement $w_{R}(t) /$ the transducer $D_{R}$.

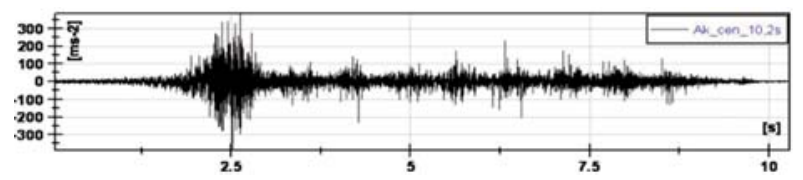

b/ Time history of the vertical rail acceleration $w^{\prime \prime}{ }_{R}(t) /$ the accelerometer $A_{R}$.

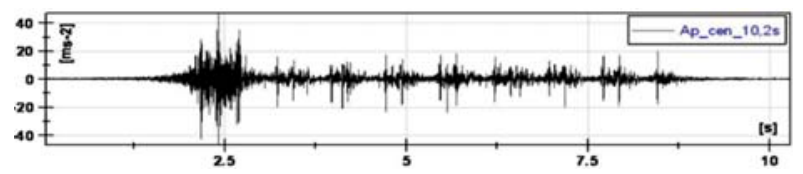

c/ Time history of the vertical sleeper acceleration $w^{\prime \prime}{ }_{S}(t) /$ the accelerometer $A_{S}$.

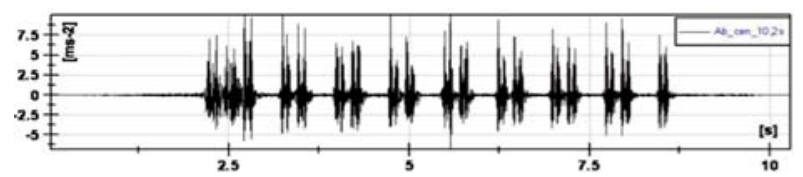

d/ Time history of the vertical ballast bed acceleration / the accelerometer $A_{B}$.

Fig. 6 Time histories of the kinematic quantities measured response $w_{R}(t), w^{\prime \prime}(t), w^{\prime \prime}{ }_{S}(t)$, for the passenger train EL 350+8 coaches, $t=6.0-12.3=6.3 \mathrm{~s}, c=119 \mathrm{~km} / \mathrm{h}$.

processes with the time-varying mean square value corresponding to the passage of bogies of coaches through the measured place. In the broader sense they may be considered as the stationary processes [8]. Then, the frequency analysis of obtained time signals can be applied as:

(1) The analysis of whole passage of the train - the measured signal is considered as a stationary one (the passage of the whole train over the measured site).

(2) The analysis of selected stationary parts of the signal and use the technique of window function (considering the short section as a stationary process). This is applied to the passage of locomotive bogies, characteristic coach bogies (couple of bogies), or the passage of characteristic parts of the train (the passage of characteristic coaches).

Then, the passage of the coach bogies over a measured place constitutes characteristic cyclic signals that in the long term they may be considered stationary, but in the short term they are nonstationary ones. The NI DIAdem interactive software for managing and analysing the measurement data [9] was applied for the spectral analyses of these signals.

\section{Spectra of the vertical acceleration of the track components due to passenger trains}

While the dominant frequency composition of the deflection $w_{x}(t)$ of rails and sleepers (measured by displacement transducers $D_{R}, D_{S}$ ) present a low frequency range only, the frequency content of acceleration $w_{x}^{\prime \prime}(t)$ of these motions gives a wide range of frequencies. At the same time we have to keep in view the relations between the displacement amplitude $\widehat{w}_{o}(f)$ and the acceleration amplitude $\widehat{w}_{o}{ }^{\prime \prime}(f)$ at a given frequency component fi.

$$
\hat{w}_{o}(f)=-\frac{\hat{w}_{o}{ }^{\prime \prime}(f)}{(2 \pi f)^{2}}
$$

Frequency analyses performed from the time signals were focused just to the frequency content of the vertical acceleration of the track component response during the passage of trains. However, for the train speed lower than the wave speeds propagating in the ground, the measured quantities may be considered as a dynamic component of the response that strongly dominates over the quasi-static axle loads response.

In the track response measurements the sampling frequency $f_{s}$ of the measured signal (the discrete sampling of a measurement time signal) plays an important role because of speedy processes (the quasi impact processes). An analysed record $\{x(t)\}$ of the total length $T_{r}$ is divided into nd segments of the length $T$ - the record length $T$. Then, the one-side auto-spectral density function $G_{X X}\left(f_{k}\right)$ for a arbitrary frequency component $f_{k}$ is given $[8,9]$ :

$$
G_{X X}\left(f_{k}\right)=\frac{2}{n_{d} T} \sum_{i=1}^{n_{d}}\left|X_{i}\left(f_{k}\right)\right|^{2}, k=1,2,3, \ldots N / 2
$$

where:

$$
X_{i}\left(f_{k}\right)=\Delta t \cdot X_{i k}=\Delta t \sum_{n=0}^{N-1} x_{i n} \cdot \exp \left[\frac{-j 2 \pi k n}{N}\right]
$$

$N$ is the sample size (the block size) $\rightarrow N=128,256,512$, 1024, 2048, 4096.

The frequency response function (FRF) $H_{X \rightarrow Y}(f)$ for an input signal $\{x(t)\}$ and output signal $\{y(t)\}$ can be displayed as the estimators:

1/ The estimator for uncorrelated output noise:

$$
H_{1, X \rightarrow Y}(f)=\frac{G_{X Y}(f)}{G_{X X}(f)}
$$

2) The estimator for uncorrelated input noise:

$$
H_{2, X \rightarrow Y}(f)=\frac{G_{Y Y}(f)}{G_{Y X}(f)}
$$

where: $G_{X Y}\left(f_{k}\right)$ is cross-spectral density function. 
The important step in the frequency analysis of the time signals is the choice of a time window function. The Hanning weighting was applied in the analyses. For the applied sample frequency $f_{s}=1000 \mathrm{~Hz}$, and a chosen sample size $N$ the resolution frequency $\beta$ is

$$
\beta=\frac{1}{T}=\frac{f_{s}}{N} \begin{array}{|c|c|c|c|c|c|}
\hline \mathrm{N} & 128 & 256 & 512 & 1024 & 2048 \\
\cline { 2 - 7 } \beta[\mathrm{Hz}] & 7.8 & 3.9 & 1.95 & 0.98 & 0.48 \\
\hline
\end{array}
$$

The Hanning weighting to "the quasi-stationary time record of the train passage" with the adequate overlapping $50 \%$ of the record length $T$ and the averaging of these signals was applied. The spectral analyses were displayed as the Power spectrum (PWS), or the Power spectral density (PSD).

\subsection{The spectral analysis of the whole train passage - analysis of a stationary signal}

The passage of the train (EL $350+8$ coaches) over the measurement site is considered and analysed as a stationary time record, as shown in Fig. 7. This approach does not take account of the non-stationary of signal.

- File TN II/No. 2-AS: Passage of the train EL 350+8 coaches, $t=6.0-12.3=6.3 \mathrm{~s}, c=32.9 \mathrm{~m} / \mathrm{s}=119 \mathrm{~km} / \mathrm{h}$, Analysis of the vertical sleeper acceleration $w_{S}^{\prime \prime}(t) /$ the accelerometer $A_{S}$, $f_{\mathrm{s}}=1000 \mathrm{~Hz}$, Fig. 7 .

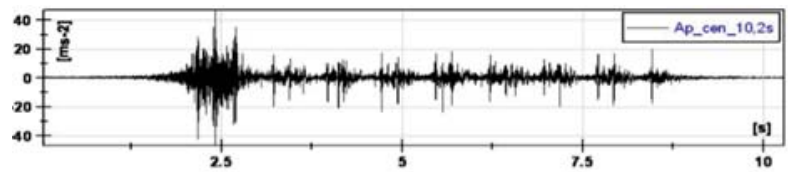

a) The time history of the vertical sleeper acceleration $w^{\prime \prime}{ }_{S}(t) /$ the accelerometer $A_{S}$

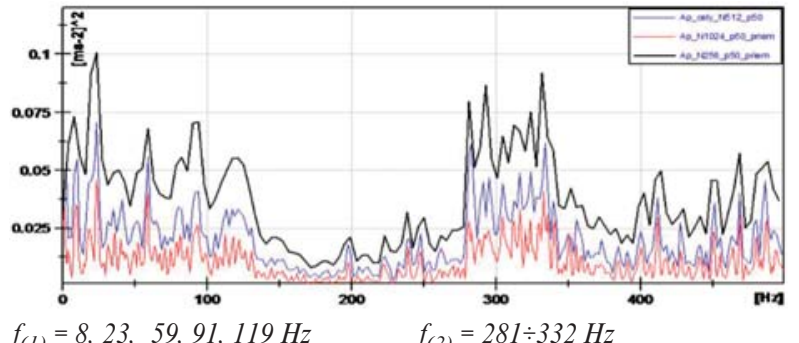

b) LABVIEW Analysis: The PWS of the sleeper acceleration $w^{\prime \prime}{ }_{S}(t) /$ the accelerometer $A_{S}$ - passage of the train EL 350+8 coaches.

The analyses for:

$\rightarrow A_{S}:$ PWS, the block size N1024, overlap 50\% - Mean 19 sp. PWS

$\rightarrow A_{S}:$ PWS, the block size N512, overlap 50\% - Mean 39 sp. PWS,

$\rightarrow A_{S}$ : PWS, the block size N256, overlap 50\% - Mean 79 sp. PWS.

The non-filtering signal for frequencies $f=(0-500 \mathrm{~Hz})$.

Fig. 7 Analysis of the vertical sleeper acceleration $w^{\prime \prime}{ }_{S}(t) /$ the accelerometer AS for the passage of the train EL 350+7 coaches, $t=6.0-12.3=6.3 \mathrm{~s}, \mathrm{c}=32.9 \mathrm{~m} / \mathrm{s}=119 \mathrm{~km} / \mathrm{h}$.
Considering to option of the block size $(N)$, option of the overlap (\%) and averaging obtained PVS the resulting mean power spectrum (PVS) gives a gross picture of frequency composition of the response. This analysis averages the frequency composition of the response.

\subsection{Spectral analysis for the locomotive EL350 passage - analysis of segmented stationary part}

The passage of locomotive EL 350 bogies over the measurement site evidently differs from the coach bogies - it is considered as a non-stationary signal with the variable mean square value. This signal can be segmented into stationary parts which can be analysed as a stationary signal, as is shown in Fig. 8.

- File TN II/No.2-A : The passage of the locomotive EL 350, $c=32.9 \mathrm{~m} / \mathrm{s}=118 \mathrm{~km} / \mathrm{h}$, Analysis of the vertical sleeper acceleration $w_{S}^{\prime \prime}(t) /$ the accelerometer $A_{S}$ - selected stationary part of the signal for $t=2.0-3.05 \mathrm{~s}=1.05 \mathrm{~s}, f_{s}=1000 \mathrm{~Hz}$, Fig. 8 .

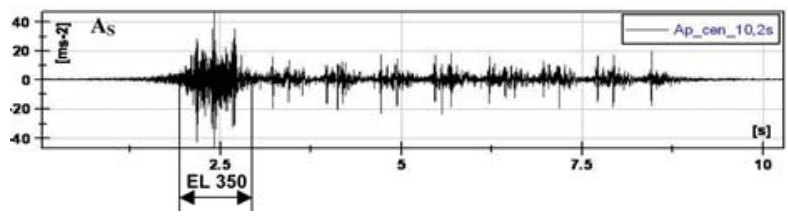

a) The time history of the vertical sleeper acceleration $w^{\prime \prime}{ }_{S}(t) /$ the accelerometer $A_{S}$ - Passage of the locomotive EL 350,

$$
t=2.0-3.05 \mathrm{~s}=1.05 \mathrm{~s} \text {. }
$$

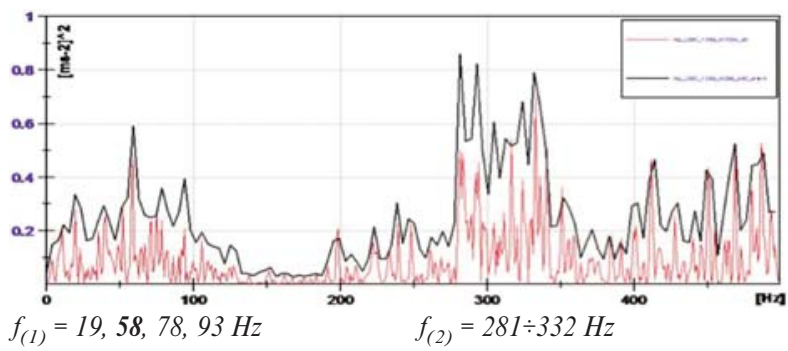

b) LABVIEW Analysis: The PWS of the sleeper acceleration $w^{\prime \prime}{ }_{S}(t) /$ the accelerometer $A_{S}$ - The passage of the locomotive EL 350,

$$
t=2.0-3,05 \mathrm{~s}=1.05 \mathrm{~s} .
$$

Analysis for: $\rightarrow A_{S}$ : PWS, the block size N1024, 1 sp. PWS,

$\rightarrow A_{S}:$ PWS, the block size N256, overlap 50\% - Mean 7 sp. PWS.

The non-filtering signal for frequencies $f=(0 \div 500 \mathrm{~Hz})$.

Fig. 8 Analysis of the vertical sleeper acceleration $w^{\prime \prime}{ }_{S}(t) /$ the accelerometer $A_{S}$ for the passage of the locomotive EL 350 - selected stationary part of the signal for $t=2.0-3.05=1.05 \mathrm{~s}$.

Comparison results of analyses in Figs. 7 and 8 show qualitative differences:

- The analysis in Fig.7 for the passage of the all train EL $350+8$ coaches, $t=0-10.24=10.24$ s gives different frequency com- 
position at the frequency area $f=0-150 \mathrm{~Hz}$ in comparison with the analysis in Fig. 8 - the passage of the locomotive EL $350, t=2.0-3.05 \mathrm{~s}=1.05 \mathrm{~s}$.

- Averaging of data for the passage of the all train EL $350+8$ coaches makes smaller values as the analysis of the locomotive L350 passage.

- The general character of the frequency composition remains approximately sustained - there are two characteristic frequency areas $f_{(1)} \approx 0-150 \mathrm{~Hz}$, and $f_{(2)} \approx 280-350 \mathrm{~Hz}$.

\subsection{Spectral analysis for the passage of coach bogies - analysis of segmented stationary part}

The passage of coach bogies over the measurement site evidently differs from the passage of the locomotive as showed the time histories, Fig.9. Then the extract part of signals can be analysed as a stationary signal (cyclic signals), as is shown in Fig. 9. The Hanning weighting to the passage of coach bogies (a quasi-stationary time record) was applied.

- File TN II/No.2-A $\mathbf{A}_{\mathbf{S}}$ The passage of coach bogies, $c=32.9 \mathrm{~m} / \mathrm{s}$ $=118 \mathrm{~km} / \mathrm{h}, t=3.01-3.8=0.79 \mathrm{~s}$, Analysis of the vertical sleeper acceleration $w_{S}^{\prime \prime}(t) /$ the accelerometer $A_{S}, f_{S}=1000 \mathrm{~Hz}$, Fig.9a, b, c.

Comparison results of analyses in Figs. 8 and 9 again show qualitative differences in the frequency composition:

- The analysis in Fig. 8 for the passage of the locomotive EL 350 $t=2.0-3.05 \mathrm{~s}=1.05 \mathrm{~s}$, gives different frequency composition than the analysis in Fig. 9 - the passage of coach bogies No.1-2, No. 2-3, No. 3-4, $t=3.01-3.8=0.79 \mathrm{~s}$

- Averaging of data for the passage of the whole train EL 350+8 coaches gives smaller values than for the passage of bogies of locomotive EL350.

- The general character of the frequency composition remains sustained - there are two characteristic frequency areas $f_{(1)} \approx$ $\approx 0 \div 150 \mathrm{~Hz}$, and $f_{(2)} \approx 280 \div 350 \mathrm{~Hz}$.

- File TN II/No.2 -Ab: a/ The time history of the vertical ballast bed acceleration $w_{B}^{\prime \prime}(t) /$ the accelerometer $A_{B}$ - The passage of coach bogies No.1-2, $t=0.79$ s, Fig. 10 a, b.

- File TN II/No.2 - $A_{S} \mid A_{B}$ : Transfer function $H_{1}\left(A_{S} \mid A_{B}\right)$ - Transfer function "sleeper $A_{S}$ " / "ballast bed $A_{B}$ " for the passage of coach bogies No. $1-2, t=3.01-3.8=0.79$ s, Fig. 11 .

\section{Results of dynamic analyses}

The measured time histories of the vertical rail acceleration $w^{\prime \prime}{ }_{R}(t)$, sleeper accelerations $w_{S}{ }_{S}(t)$, and ballast bed acceleration $w^{\prime \prime}{ }_{B}(t)$ and corresponding spectra PSD or PWS give the principal picture of the dynamic behaviour of the track structure for passages of the characteristic passenger trains.

- The amplitudes of the vertical acceleration of track components the rail $w_{R}^{\prime \prime}(t), w_{S}{ }_{S}(t)$, and $\left.w_{B}{ }_{B}(t)\right)$ are gradually damped in the

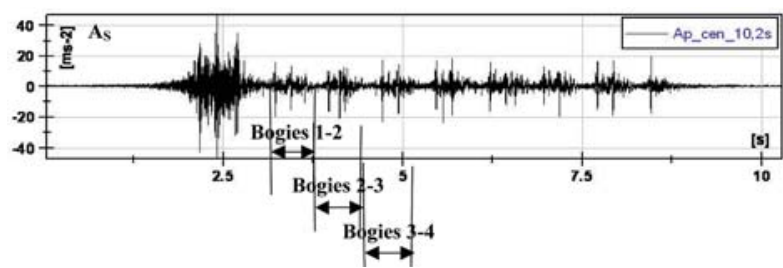

a) The time history of the vertical sleeper acceleration $w^{\prime \prime}{ }_{S}(t) /$ the accelerometer $A_{S}$-The passage of coach bogies No.1-2, No. 2-3, No. $3-4, t=3.01-3.8=0.79 \mathrm{~s}$

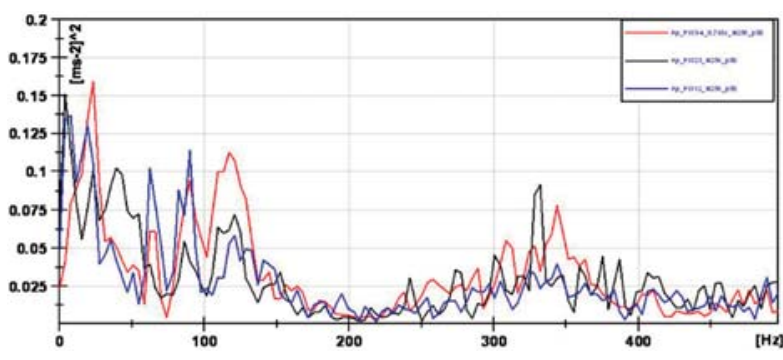

b) LABVIEW Analysis: The PWS of the sleeper acceleration $w^{\prime \prime}(t) /$ the accelerometer $A_{S}$ - The passage of coach bogies No. 1-2, No. 2-3, No. 3-4, $t=3.01-3.8=0.79$ s. Analysis for bogies No.1-2, bogies No.2-3, bogies No.3-4, $\rightarrow$ the block size N256, overlap 50\% - the mean of 5 PWS. The non-filtering signal for frequencies $f=(0 \div 500 \mathrm{~Hz})$

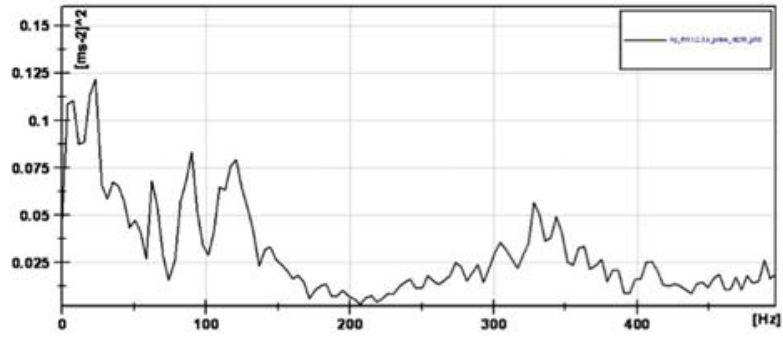

c) b) LABVIEW Analysis: The PWS of the sleeper acceleration $w^{\prime \prime}{ }_{S}(t) /$ the accelerometer $A_{S}$ - The passage of coach bogies No.1-2, No. 2-3,

No. 3-4, $t=3.01-3.8=0.79$ s. The mean of $3 \mathrm{PWS}$, for the three bogies passage: bogies No. 1-2, bogies No. 2-3, bogies No. 3-4. The nonfiltering signal for frequencies $f=(0 \div 500 \mathrm{~Hz})$.

Fig. 9 Analysis of the vertical sleeper acceleration $w^{\prime \prime}{ }_{S}(t) /$ the accelerometer AS for the passage of coach bogies No.1-2, No. 2-3, No. 3-4,

$$
t=3.01-3.8=0.79 s \text {. }
$$

vertical direction. The standard acceleration rail peaks for locomotives occur up to $300 \mathrm{~m} / \mathrm{s}^{2}$ and for coaches up to $50 \div 100$ $\mathrm{m} / \mathrm{s}^{2}$. These peaks are reduced on the sleepers up to $30 \mathrm{~m} / \mathrm{s}^{2}$ and on the ballast bed to $8 \div 10 \mathrm{~m} / \mathrm{s}^{2}$, as can be seen in Fig. 6 .

- Spectral analysis of segmented stationary parts of the response

The passage of vehicle bogies generally represent nonstationary random processes with the time-varying mean square values corresponding to the locomotive bogie passages and coach bogie passages. The analysis of a selected stationary part of the signal considered as a stationary process applied on the passage of loco- 

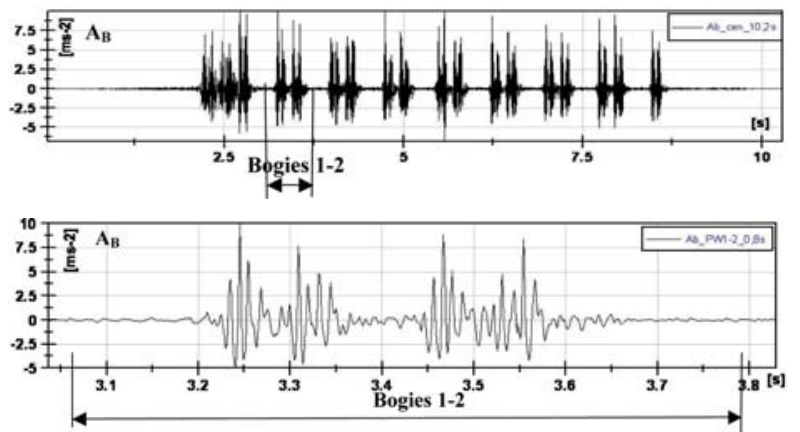

a) The time history of the vertical ballast bed acceleration $w_{B}^{\prime \prime}(t) /$ the accelerometer $A_{B}$-The passage of coach bogies No. 1-2, $t=3.01-3.8=0.79 \mathrm{~s}$.

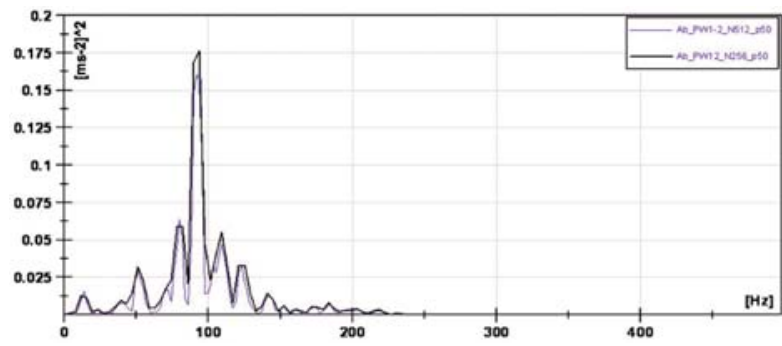

$f_{(I)}=13,51,92,123 \mathrm{~Hz}$

b) LABVIEW Analysis: The PWS of the ballast bed acceleration $w^{\prime \prime}(t)$ the accelerometer $A_{B}$, the passage of coach bogies No.1-2, $t=3.01-3.8=0.79 \mathrm{~s}$.

$\rightarrow$ the block size N512, overlap 50\% - The mean of $2 \mathrm{sp.} \mathrm{PWS,}$

$\rightarrow$ the block size N256, overlap 50\% - The mean of $5 \mathrm{sp}$. PWS. The non-filtering signal for frequencies $f=(0 \div 500 \mathrm{~Hz})$.

Fig. 10 Analysis of the vertical ballast bed acceleration $w^{\prime \prime}{ }_{B}(t) /$ the accelerometer $A_{B}$ for the passage of coach bogies No. 1-2,

$$
t=3.01-3.8=0.79 \mathrm{~s} \text {. }
$$

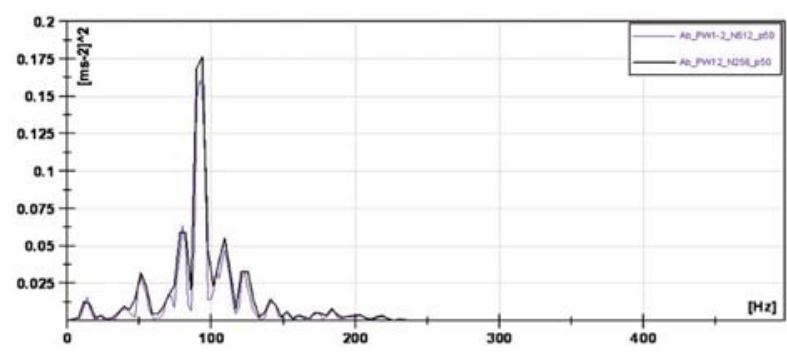

$$
f_{(I)}=13,39,55,78,95,106 \mathrm{~Hz} \quad f_{(1)}=171,178,201,219 \mathrm{~Hz}
$$

Fig. 11 LABVIEW Analysis: Transfer function $\boldsymbol{H}_{\boldsymbol{1}}\left(\boldsymbol{A}_{\boldsymbol{S}} / \boldsymbol{A}_{\boldsymbol{B}}\right)$ - The passage of coach bogies No.1-2, $t=3.01-3.8=0.79 \mathrm{~s} \rightarrow$ the block size N512, overlap 50\% - The mean of $2 \mathrm{sp}$. PWS.

The non-filtering signal for frequencies $f=(0 \div 500 \mathrm{~Hz})$. motive bogies, characteristic coach bogies (couple of bogies), or the passage of characteristic parts of the train (the passage of characteristic coaches) is analysed by the time window technique. The spectral composition corresponding to passages of bogies may be evaluated.

The frequency content of the vertical acceleration on the track components - rails, sleepers, and ballast bed differs:

- The frequency spectrum for a rail acceleration is a broadband spectrum in the frequency range $f=0-500 \mathrm{~Hz}$, without any sharp spectral components. The frequency component content at the frequency area $f \approx(0 \div 150 \mathrm{~Hz})$ has the dominant signification on the rail response.

- The frequency spectrum for a sleeper acceleration is a broadband spectrum too, but two marked frequency areas be detected: $f_{(1)} \approx 0 \div 100 \mathrm{~Hz}$, and $f_{(2)} \approx 280 \div 320 \mathrm{~Hz}$. They present two characteristic damped resonant areas occurring in all the measured signals:

a/ In the first area the frequency range $f_{(1)}=(55 \div 90 \mathrm{~Hz})$ with a max. $f_{(1)} \approx 60 \mathrm{~Hz}$ is dominant - the rails, sleepers, and ballast layer vibrate together and constitute dominant effect on the sleeper response.

b/ In the second one the frequency range $f_{(2)}=(280 \div 320 \mathrm{~Hz})$ with a $\max . f_{(2)} \approx 280 \mathrm{~Hz}$ is dominant - the rails vibrate on sleepers.

- The frequency spectrum for ballast bed is narrow-band and always is concentrated about frequencies $f=80 \div 120 \mathrm{~Hz}$.

The presented spectra of vertical accelerations of track components can be considered as characteristic, occurring in all the analysed passages of the passenger trains, because the dynamic loading is similar. The characteristic and important spectral components are appeared in the frequency range $f=(0 \div 150 \mathrm{~Hz})$ in all the track components when the rails, sleepers, and ballast bed vibrate together. A gradual damping of frequency components in the vertical direction is apparent in all spectral analyses in Figs. $7 \div 10$.

- The spectra corresponding to passages of heavy freight trains are different in comparison of passenger trains, because the frequency of loading, the speed of freight trains, the mass of carriages are different from passenger trains, and a quasi-static preloaded of the track is higher.

- The comparison of the measured amplitudes of the vertical acceleration of track components (rails, sleepers and the ballast bed) indicates the proper damping and filtration of the frequency components at the transmission of dynamic loading from the rail to the substructure and to the subgrade as the consequence of the resilient rail fastening (Vossloh Skl 14), the resilient pads, and the generally proper function ballast bed and subgrade.

- A very negative impact of coaches with some defective wheels (the out-of-round wheels) to the vertical track acceleration was indicated. Peaks reached up to a double higher value of the response than the coaches with smooth wheels. 


\section{Conclusions}

Dynamic behaviour of the track structure during the passage of passenger trains was investigated as the vertical acceleration of the track components - the rails $w_{R}^{\prime \prime}(t)$, the sleepers $w_{S}^{\prime \prime}(t)$, and the ballast layer $w^{\prime \prime}{ }_{B}(t)$ at the depth of placing sleepers. The frequency analysis was made for the mid frequency range $f=(0 \div$ $500 \mathrm{~Hz}$ ). Measured signals include a large number of excitation sources - geometrical errors, or irregularity on the rails, wheel out-of-roundness, irregular track stiffness, etc. The selected stationary part of the measured signals considered as a stationary process (the passage of locomotive bogies, characteristic coach bogies or couple of bogies, the passage of characteristic coaches) were analysed by the time window technique. The spectrum changes corresponding to characteristic bogie passages were evaluated.
The presented experimental analyses for the new construction of the track structure are complementary to the other data sets the measurement of the passage of freight trains, or the measurement of the response on other types of ballasted tracks. Frequency analyses of time histories of the vertical rail acceleration $w_{R}{ }_{R}(t)$, the sleeper accelerations $w_{S}{ }_{S}(t)$, and the ballast bed acceleration $w^{\prime \prime}{ }_{B}(t)$ provide the principal picture of the dynamic behaviour of the track structure, the transmission and the damping of frequency components through the track components under the passage of the characteristic passenger trains. Although they moderately differ under the passage of different trains the main features and the character of the response is maintained.

\section{References}

[1] KNOTHE, K., GRASSIE, S.: Modelling of Railway Track and Vehicle/Track Interaction at High Frequencies. Vehicle System Dynamic, Nr. 22, 1993, pp. 209-262.

[2] KNOTHE, K., WU, Y.: Receptance Behaviour of Railway Track and Subgrade. Archive of applied Mechanics, Nr. 68, 1998, Spring Verlag, pp. 457-470.

[3] DEGRANDE, G.: Free Field Vibration Measurements During the Passage of Thalys High Speed Train. Report BWM-2000-06, Dept. of Civil Eng. KU Leuven.

[4] SHEN-HAW JU, HUNG-TA LIN, JENG-YUAN HUANG: Dominant Frequencies of Train-induced Vibrations, J. of Sound and Vibration, Nr. 319, 2009, pp. 247-259.

[5] SICAR, M.: Vehicle-track Interaction Concentrated to the Track Response (in Slovak). PhD Thesis, University of Zilina, p. $180,1996$.

[6] MORAVCIK, M.: Track Mechanics - Parts 1, 2. - Theoretical Analysis and Simulation Track Mechanics Problems (in Slovak), EDIS, Žilina 2002. Part 1, ISBN 80-7100-983-0, 300 p., Part 2, ISBN 80-7100-984-9, p. 312.

[7] MORAVCIK, M.: Dynamic Behaviour of Railway Track - Experimental Measurements. Communication - Scientific Letters of the University of Zilina, Nr. 3, 2002, ISSN 1335-4205, pp. 45-62.

[8] BENDAT J., PIERSOL A.: Random Data - Analysis and Measurement Procedures. John Wiley and Sons, 1986, ISBN 0-471-040002, $566 \mathrm{p}$.

[9] DIAdem/NT Manual: Advance Course Manual, Versiom 10.0, 2006. 